\title{
Goodput Enhancement of IEEE 802.11a Wireless LAN via Link Adaptation
}

\author{
Daji Qiao \\ Real-Time Computing Laboratory \\ The University of Michigan \\ Email: dqiao@eecs.umich.edu
}

\begin{abstract}
IEEE 802.11a is a new high-speed physical layer (PHY) defined for the $5 \mathrm{GHz}$ U-NII bands as a supplement to the existing IEEE 802.11 wireless LAN (WLAN) standard. In this paper, we give an overview of the IEEE 802.11a Orthogonal Frequency Domain Multiplexing (OFDM) PHY with eight different PHY rates as well as the distributed coordination function (DCF) of the IEEE 802.11 MAC, then derive the goodput performance analytically for peer-to-peer communication under the DCF. Based on the numerical results, we claim that link adaptation, which performs both dynamic fragmentation and PHY rate selection depending on the wireless channel condition between the transmitter and receiver, is an attractive way to improve the goodput performance of an IEEE 802.11a wireless LAN. Finally, we propose a system architecture to perform link adaptation.
\end{abstract}

\section{INTRODUCTION}

The IEEE 802.11 standard [1] is the first international standard for wireless local area networks (WLANs), which defines a medium access control (MAC) sublayer, MAC management protocols and services, and three low-speed physical layers (PHYs) operating in both 1 and 2 Mbps. Recently, the increasing number of wireless users and the growing demand of high-speed multimedia services have led to the requirement of higher data rates and bandwidths to be supported by future wireless systems.

One new high-speed physical layer, the IEEE 802.11a PHY [2], has been developed to extend the existing IEEE 802.11 standard in the $5 \mathrm{GHz}$ U-NII bands. It is an Orthogonal Frequency Domain Multiplexing (OFDM) radio, which provides eight different $\mathrm{PHY}$ modes with data rates ranging from $6 \mathrm{Mbps}$ up to 54 Mbps. In addition to the use of the advanced modulation schemes compared to the existing low-speed PHYs, another important aspect of the IEEE 802.11a PHY is that, a convolutional code of rate $1 / 2$ is included as part of the physical layer to improve the frame transmission reliability. However, the IEEE 802.11 a PHY has to work under the existing MAC protocol that was originally designed for low-speed PHYs. Therefore, it is important to add the link adaptation capability to the existing MAC protocol, so that the proper PHY mode as well as the best MAC frame size can be adaptively selected to combat the variation of the wireless medium condition, hence improving the goodput performance of an IEEE 802.11a wireless LAN. Here, the goodput refers to the bandwidth the user actually receives after all the overheads are accounted for, including the MAC overhead, the PHY overhead, and the retransmission overhead.

In [3], the goodput performance was analyzed for the IEEE 802.11 wireless LAN using Lucent Technologies' WaveLAN radio. However, since the high-speed OFDM PHY was not available at that time, the authors assumed the QPSK modulation without forward error correction (FEC), and analyzed the goodput performance for different size of the MAC service data units (MSDUs), which are generated by the IEEE 802.2 Logi-

The work reported in this paper was supported in part by AFOSR under Grant No. F49620-00-1-0327.

\author{
Sunghyun Choi \\ Video Communications Department \\ Philips Research - USA \\ Email: sunghyun.choi@philips.com
}

cal Link Control (LLC) sublayer. Actually, as indicated in the IEEE 802.11 standard, an MSDU can be further fragmented into smaller MAC frames, i.e., MAC protocol data units (MPDUs), for transmission. In this paper, we explore the following two adaptive techniques: dynamic fragmentation of MSDUs, and dynamic PHY mode selection for each MPDU transmission. Our link adaptation idea is to select the optimal combination of the PHY mode and the fragment size, depending on the wireless channel condition, to achieve the best goodput performance.

The rest of this paper is organized as follows. Section 2 introduces the distributed coordination function (DCF) of the IEEE 802.11 MAC and the IEEE 802.11a high-speed OFDM PHY. The error probability analysis and the goodput performance analysis are presented in Section 3 and Section 4, respectively. Section 5 presents and discusses the numerical results, and proposes a system architecture to adopt link adaptation. Finally, this paper concludes with Section 6.

\section{SYSTEM OVERVIEW}

The IEEE 802.11 MAC sublayer provides a fairly controlled access to the shared wireless medium through two different access mechanisms: the basic access mechanism, called the distributed coordination function (DCF), and a centrally controlled access mechanism, called the point coordination function (PCF). In this paper, we are focusing on the goodput performance of the IEEE 802.11 wireless LAN under the DCF, which will be discussed in details in Section II-A. The goodput performance analysis for the PCF will be treated in a separate paper.

The PHY is the interface between the MAC and the wireless medium, which transmits and receives data frames over the shared wireless medium. The frame exchange between MAC and PHY is under the control of the physical layer convergence procedure (PLCP) sublayer.

\section{A. DCF of IEEE 802.11 MAC}

The basic access mechanism of the IEEE 802.11 MAC, called the DCF, achieves automatic medium sharing between compatible PHYs through the use of carrier sense multiple access with collision avoidance (CSMA/CA) with random backoff. Before a station starts transmission, it shall sense the wireless medium to determine if another station is transmitting. If the medium is idle, the transmission may proceed. If the medium is busy, the station shall defer until the end of the current transmission. The CSMA/CA mechanism requires that a gap of a minimum specified space exist between contiguous frame transmissions. A station shall ensure that the medium has been idle for the specified inter-frame space before attempting to transmit.

The distributed inter-frame space (DIFS) is used by stations 
operating under the DCF to transmit data frames and management frames. A station using the DCF has to follow two medium access rules: (1) the station shall be allowed to transmit only if its carrier-sense mechanism determines that the medium has been idle for at least DIFS time; and (2) in order to reduce the collision probability between multiple stations accessing the wireless medium, the station shall select a random backoff interval after deferral or prior to attempting to transmit again immediately after a successful transmission.

One important characteristics of the IEEE 802.11 MAC is that an acknowledgment (ACK) frame shall be transmitted by the receiver upon a successful data frame reception. Only after receiving an ACK frame correctly, the transmitter assumes that the data frame was delivered successfully. The short inter-frame space (SIFS), which is smaller than DIFS, is the time space between a data frame reception and the ACK frame transmission. Using this smallest gap between transmissions within the frame exchange sequence prevents other stations, which are required to wait for the medium to be idle for a longer gap, e.g., at least DIFS time, from attempting to use the medium, thus giving priority to completion of the frame exchange sequence in progress.

The process of partitioning an MSDU into smaller MAC frames is called fragmentation. Fragmentation creates MPDUs smaller than the original MSDU length to improve reliability, by increasing the probability of successful fragment transmissions. Each fragment is sent as an independent transmission and is acknowledged separately. Once a station has contended for the medium, it shall continue to send fragments with a SIFS gap between the ACK reception and the start of the subsequent fragment transmission until either all the fragments of a single MSDU have been sent, or an ACK frame is not received. The process of sending multiple fragments after contending for the medium is called a fragment burst. However, if any fragment transmission fails, the station shall attempt to retransmit the failed fragment after the backoff procedure. The different timings of successful fragment transmission and fragment transmission failure are shown in Figs. 1 and 2, respectively.

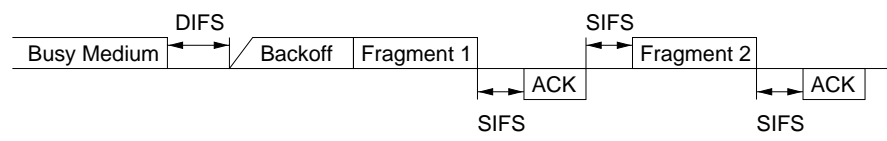

Fig. 1. Timing of successful fragment transmission

\begin{tabular}{|c|c|c|c|c|}
\hline & DIFS & ACK Timeout & & \\
\hline Busy Medium & $\hookrightarrow$ Backoff & Fraghent $1 \hookrightarrow$ Backoff & Fragment 1 & \\
\hline
\end{tabular}

Fig. 2. Timing of fragment transmission failure and retransmission

Based on the above discussions, we notice that a transmission cycle under the DCF consists of the following phases: the DIFS/SIFS deferral phase, the backoff/contention phase if necessary, the data/fragment transmission phase, the SIFS deferral phase, and the ACK transmission phase.

\section{B. IEEE 802.11a OFDM PHY}

The OFDM has been selected as the modulation scheme for the IEEE 802.11a PHY, which is very similar to the modulation scheme adopted in Europe for HIPERLAN/2 PHY [4]. A key feature of the IEEE 802.11a PHY is to provide eight PHY modes with different modulation schemes and different coding rates, which makes the idea of link adaptation feasible. As listed in Table I, the OFDM system provides a wireless LAN with capabilities of communicating at 6 to $54 \mathrm{Mbps}$. Forward error correction is performed by bit interleaving and rate- $1 / 2$ convolutional coding. The higher code rates of $2 / 3$ and $3 / 4$ are obtained by puncturing the original rate- $1 / 2$ code.

\begin{tabular}{|c|c|c|c|c|}
\hline Mode & Modulation & Code Rate & Data Rate & BpS \\
\hline \hline 1 & BPSK & $1 / 2$ & $6 \mathrm{Mbps}$ & 3 \\
\hline 2 & BPSK & $3 / 4$ & $9 \mathrm{Mbps}$ & 4.5 \\
\hline 3 & QPSK & $1 / 2$ & $12 \mathrm{Mbps}$ & 6 \\
\hline 4 & QPSK & $3 / 4$ & $18 \mathrm{Mbps}$ & 9 \\
\hline 5 & $16-\mathrm{QAM}$ & $1 / 2$ & $24 \mathrm{Mbps}$ & 12 \\
\hline 6 & $16-\mathrm{QAM}$ & $3 / 4$ & $36 \mathrm{Mbps}$ & 18 \\
\hline 7 & 64-QAM & $2 / 3$ & $48 \mathrm{Mbps}$ & 24 \\
\hline 8 & 64-QAM & $3 / 4$ & $54 \mathrm{Mbps}$ & 27 \\
\hline
\end{tabular}

TABLE I

EIGHT PHY MODES OF IEEE 802.11A PHY

During the transmission, the MPDUs from the MAC sublayer shall be provided with a PLCP preamble and a PLCP header to create PLCP protocol data units (PPDUs).

\section{ERRor PROBABILITy ANALYSIS}

In this paper, we assume that the noise over the wireless medium is white Gaussian with spectral density $N_{0} / 2$. Although this additive white Gaussian noise channel (AWGN) model is not a realistic assumption, we believe that our goodput analysis based on the AWGN model will show the same trend with that based on a more realistic and complicated channel model, so that the results presented in this paper can be a good guidance for the reality.

\section{A. Bit Error Probability}

The symbol error probability for an $M$-ary QAM modulation [5] with $M=4,16,64$ can be calculated by

$$
P_{M}=1-\left(1-P_{\sqrt{M}}\right)^{2},
$$

where

$$
P_{\sqrt{M}}=2 \cdot\left(1-\frac{1}{\sqrt{M}}\right) \cdot Q\left(\sqrt{\frac{3}{M-1} \cdot \frac{E_{a v}}{N_{0}}}\right)
$$

is the symbol error probability for the $\sqrt{M}$-ary PAM modulation with the average signal-to-noise ratio (SNR) per symbol, $E_{a v} / N_{o}$. With a Gray coding, the bit error probability for an $M$-ary QAM modulation can be approximated by

$$
P_{b}^{(M)} \approx \frac{1}{\log _{2} M} \cdot P_{M}
$$

Note that 4-ary QAM and QPSK are identical. For BPSK modulation, the bit error probability is the same as the symbol error probability, which is given by

$$
P_{b}^{(2)}=P_{2}=Q\left(\sqrt{\frac{2 E_{a v}}{N_{0}}}\right) .
$$




\section{B. Packet Error Probability}

In [6], an upper bound was given on the packet error probability, under the assumption of binary convolutional coding and hard-decision Viterbi decoding with independent errors at the channel input. For an $L$-octet long packet to be transmitted using PHY mode $m(1 \leq m \leq 8)$, this bound is

$$
P_{e}^{m}(L) \leq 1-\left(1-P_{u}^{m}\right)^{8 L},
$$

where the union bound $P_{u}^{m}$ of the first-event error probability is given by

$$
P_{u}^{m}=\sum_{d=d_{\text {free }}}^{\infty} a_{d} \cdot P_{d},
$$

where $d_{f r e e}$ is the free distance of the convolutional code selected in PHY mode $m, a_{d}$ is the total number of error events of weight $d$, and $P_{d}$ is the probability that an incorrect path at distance $d$ from the correct path being chosen by the Viterbi decoder. When hard decision decoding is applied, $P_{d}$ is given by

$$
P_{d}= \begin{cases}\sum_{k=(d+1) / 2}^{d}\left(\begin{array}{l}
d \\
k
\end{array}\right) \cdot \rho^{k} \cdot(1-\rho)^{d-k}, & \text { if } d \text { is odd } \\
\frac{1}{2} \cdot\left(\begin{array}{c}
d \\
d / 2
\end{array}\right) \cdot \rho^{d / 2} \cdot(1-\rho)^{d / 2} & \\
\quad+\sum_{k=d / 2+1}^{d}\left(\begin{array}{l}
d \\
k
\end{array}\right) \cdot \rho^{k} \cdot(1-\rho)^{d-k}, & \text { if } d \text { is even }\end{cases}
$$

where $\rho$ is the bit error probability for the modulation scheme selected in PHY mode $m$, and is given by Eq. (3) or (4). The value of $a_{d}$ can be obtained either from the transfer function or by a numerical search [7].

\section{GoOdPut PERFormanCE}

Recall that the transmission cycle under the DCF of the IEEE 802.11 MAC consists of the following phases, which are executed repetitively: the DIFS/SIFS deferral phase, the backoff/contention phase if necessary, the data/fragment transmission phase, the SIFS deferral phase, and the ACK transmission phase. The related characteristics for the IEEE 802.11a PHY are listed in Table II, where some of them will be discussed in details later. In this section, we are going to state the assumptions to be used for our goodput analysis, discuss the MAC/PHY/retransmission overheads, and analyze the goodput performance of an IEEE 802.11a wireless LAN.

\section{A. Assumptions}

In general, it is difficult, if not impossible, to analyze the goodput performance for a real IEEE 802.11 wireless LAN system, where multiple stations are contending for the shared wireless medium. However, in this paper, since the focus is on the trend of MAC/PHY/retransmission overheads affecting the goodput performance, we make the following assumptions to simplify our analysis.

Assume that two stations running the DCF are communicating with each other with no interfering stations nearby. Only one station is transmitting and its queue is never empty. Therefore, there are no collisions on the wireless medium, but it is also never idle for any longer period than required by the DCF medium access rules. In addition, we assume that there is no retry limit for each frame. We assume that there is no power control on the transmitting station, i.e., each OFDM symbol is transmitted at the same power level. Besides, we neglect the air propagation delays in our goodput analysis. Finally, we assume that the ACK frame is transmitted at the same rate as the data frame which it is acknowledging.

\section{B. MAC/PHY Layer Overheads}

\begin{tabular}{|c|c|c|c|c|c|c|c|}
\hline $\begin{array}{r}\text { octets: } 2 \\
\begin{array}{r}\text { Frame } \\
\text { Control }\end{array}\end{array}$ & $\begin{array}{c}\text { Duration/ } \\
\text { ID }\end{array}$ & Address 1 & Address 2 & Address 3 & $\begin{array}{c}\text { Sequence } \\
\text { Control }\end{array}$ & Frame Body & FCS \\
\hline
\end{tabular}

Fig. 3. Frame format of a data-type MPDU

In the IEEE 802.11 MAC, each data-type MPDU consists of the following basic components, as shown in Fig. 3: a $M A C$ header, a variable length information frame body, and a frame check sequence (FCS). All the fields except the frame body, which is 28 octets in total, contribute to the MAC overhead for a data/fragment frame. Fig. 4 illustrates the frame format of an ACK frame MPDU.

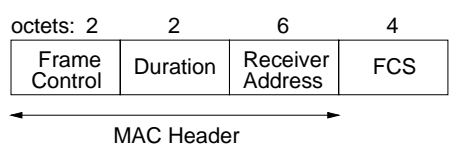

Fig. 4. Frame format of an ACK frame MPDU

The PPDU format of the IEEE 802.11a PHY is shown in Fig. 5, which includes PLCP preamble, PLCP header, MPDU, tail bits, and pad bits. The PLCP preamble field, with the duration of $t$ PLCPPreamble, is composed of 10 repetitions of a short training sequence $(0.8 \mu \mathrm{s})$ and 2 repetitions of a long training sequence $(4 \mu s)$. The PLCP header except the SERVICE field, with the duration of $t P L C P H e a d e r$, constitutes a separate OFDM symbol, which is transmitted with BPSK modulation and the rate- $1 / 2$ convolutional coding. The 6 "zero" tail bits are used to return the convolutional codec to the "zero state", and the pad bits are used to make the resulting bit string to be a multiple of OFDM symbols. Each OFDM symbol interval, denoted by $t$ Symbol, is $4 \mu s$. The 16-bit SERVICE field of the PLCP header and the MPDU (along with 6 tail bits and pad bits), represented by DATA, are transmitted at the data rate specified in the RATE field.

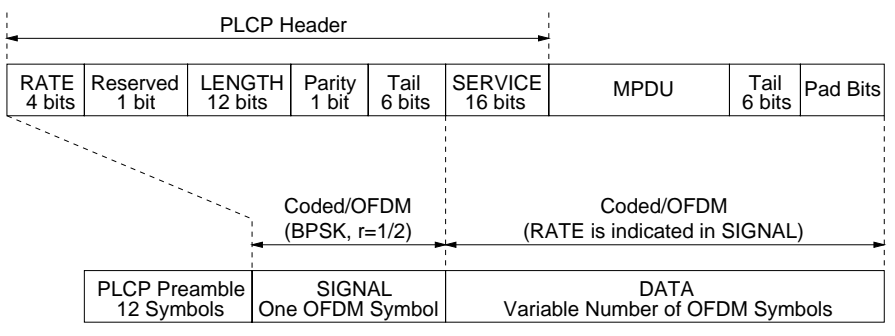

Fig. 5. PPDU frame format of IEEE 802.11a OFDM PHY

Based on the above analysis, for $L$-octet long information to be transmitted over the IEEE 802.11 a physical layer using PHY mode $m$, the transmission duration is

$$
\begin{aligned}
T_{\text {data }}^{m}(L) & =t P L C P \text { Preamble }+ \text { tPLCPHeader } \\
& +\left\lceil\frac{30.75+L}{B p S(m)}\right\rceil \cdot \text { Symbol. }
\end{aligned}
$$




\begin{tabular}{|l|l|l|}
\hline Characteristics & Value & Comments \\
\hline \hline aSlotTime & $9 \mu \mathrm{s}$ & slot time \\
\hline aSIFSTime & $16 \mu \mathrm{s}$ & SIFS time \\
\hline aDIFSTime & $34 \mu \mathrm{s}$ & aDIFSTime $=$ aSIFSTime $+2 \times$ aSlotTime \\
\hline aCWmin & 15 & min contention window size in unit of aSlotTime \\
\hline aCWmax & 1023 & max contention window size in unit of aSlotTime \\
\hline tPLCPPreamble & $16 \mu s$ & PLCP preamble duration \\
\hline tPLCPHeader & $4 \mu s$ & PLCP header duration except the SERVICE field \\
\hline$t$ Symbol & $4 \mu s$ & OFDM symbol interval \\
\hline
\end{tabular}

TABLE II

IEEE 802.11 A OFDM PHY CHARACTERISTICS

Note that the Bytes-per-Symbol information for PHY mode $m$, $B p S(m)$, is given in Table I. Similarly, the transmission duration for an ACK frame using PHY mode $m$ is

$$
\begin{aligned}
T_{a c k}^{m} & =t \text { PLCPPreamble }+t \text { PLCPHeader } \\
& +\left\lceil\frac{16.75}{B p S(m)}\right\rceil \cdot \text { S Symbol }
\end{aligned}
$$

\section{Backoff/Contention Phase}

The random backoff interval is in the unit of aSlotTime, and this random integer is drawn from a uniform distribution over the interval $[0, C W]$, where $C W$ is the contention window parameter. $C W$ shall take an initial value of $a C W m i n$. In the case of an unsuccessful transmission, $C W$ is updated to $[2 \times(C W+1)$ 1]. Once $C W$ reaches $a C W \max$, it shall remain at the value of aCWmax until it is reset. In the case of a successful acknowledged transmission, the $C W$ value is reset to $a C W \min$ before the random backoff interval is selected.

Let $\bar{T}_{b k o f f}(i)$ denote the average backoff interval after $i$ consecutive unsuccessful transmission attempts, and it can be calculated by

$$
\bar{T}_{b k o f f}(i)= \begin{cases}\frac{2^{i} \cdot(a C W \min +1)-1}{2} \cdot \text { aSlotTime }, & 0 \leq i \leq 6, \\ \frac{a C W \max }{2} \cdot a \text { SlotTime }, & i \geq 6 .\end{cases}
$$

\section{Goodput Analysis}

Assume that $L$-octet long information is to be transmitted using PHY mode $m$. Then, the probability of a successful transmission cycle can be calculated by

$$
P_{\text {good_cycle }}^{m}(L)=\left(1-P_{e, \text { data }}^{m}(L)\right) \cdot\left(1-P_{e, a c k}^{m}\right),
$$

where $P_{e, \text { data }}^{m}(L)$ and $P_{e, a c k}^{m}$ are the data/fragment transmission error probability and the ACK transmission error probability, respectively. Since the data/fragment frame is normally much longer than the ACK frame, the possibility is tiny for the ACK frame to be lost while the data/fragment frame gets through. Therefore, we have the approximation of

$$
P_{\text {good_cycle }}^{m}(L) \approx 1-P_{e, d a t a}^{m}(L)
$$

where

$$
P_{e, d a t a}^{m}(L)=1-\left(1-P_{e}^{1}(3)\right) \cdot\left(1-P_{e}^{m}(30.75+L)\right) .
$$

Here, $P_{e}^{1}(3)$ is the packet error probability of PLCP header, because PLCP header is 24-bit long and always transmitted with
BPSK modulation and rate-1/2 convolutional coding, i.e., PHY mode 1 . The calculations of $P_{e}^{1}(\cdot)$ and $P_{e}^{m}(\cdot)$ are given by Eq. (5).

Let $\mathcal{N}_{\text {frag }}$ and $n$ be the number of fragments and the number of consecutive unsuccessful transmission attempts before the current transmission, respectively. Since we assume that there is no retry limit for each frame, the contention window parameter $C W$ will always be reset to $a C W m i n$ after the successful transmission of the last fragment of a fragment burst. Therefore, the average time space between contiguous MSDU transmissions is

$$
\mathcal{O}_{m s d u}=a D I F S T i m e+\bar{T}_{b k o f f}(0) .
$$

By referring to Fig. 1, each successful fragment transmission duration is equal to the fragment transmission time, plus the ACK transmission time, and plus two SIFS times. However, whenever the fragment transmission fails, the station has to wait for an ACK timeout period and then execute a backoff procedure before the fragment retransmission (see Fig. 2). According to the Specification and Description Language formal description of the IEEE 802.11 MAC operation [1], an ACK timeout period is equal to a SIFS time, plus the ACK transmission time, and plus a slot time. Therefore, the average transmission duration for each fragment, $\mathcal{D}_{\text {frag }}$, can be calculated by Eq. (15) (on next page), where $T_{\text {data }}^{m}(L), T_{a c k}^{m}$, and $\bar{T}_{b k o f f}(i)$ are given by Eq. (8), (9), and (10), respectively, and

$$
P[n=i]=\left[1-P_{\text {good_cycle }}^{m}\left(\frac{L}{\mathcal{N}_{\text {frag }}}\right)\right]^{i} \cdot P_{\text {good_cycle }}^{m}\left(\frac{L}{\mathcal{N}_{\text {frag }}}\right) .
$$

The average goodput can then be approximated by

$$
\mathcal{G}(\text { Mbps })=\frac{8 \cdot L}{\mathcal{O}_{\text {msdu }}+\mathcal{N}_{\text {frag }} \cdot \mathcal{D}_{\text {frag }}-\text { aSIFSTime }},
$$

where the-aSIFSTime term is due to the fact that the first fragment of a fragment burst will be transmitted right after the backoff interval without an additional SIFS deferral.

\section{NumericAl RESUlts AND Discussion}

According to the IEEE 802.11 standard, the length of an MSDU must be less than or equal to 2304 octets. The length of a fragment MPDU shall be an equal even number of octets for all fragments except the last one of a fragment burst, which may be smaller. Besides, by referring to the Specification and Description Language formal description of the IEEE 802.11 MAC operation [1], the minimum fragment size is 228 octets. 
$\mathcal{D}_{\text {frag }}=\sum_{i=1}^{\infty} P[n=i] \cdot \sum_{j=1}^{i}\left[a S I F S T i m e+T_{a c k}^{m}+a \operatorname{SlotTime}+\bar{T}_{\text {bkoff }}(j)+T_{\text {data }}^{m}\left(\frac{L}{\mathcal{N}_{\text {frag }}}\right)\right]+2 \cdot a S I F S T i m e+T_{\text {data }}^{m}\left(\frac{L}{\mathcal{N}_{\text {frag }}}\right)+T_{\text {ack }}^{m}$

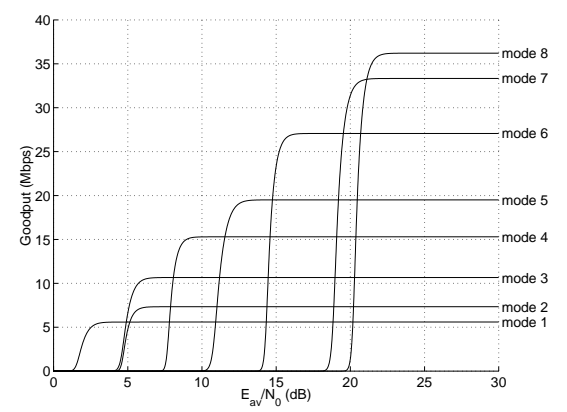

(a) no fragmentation

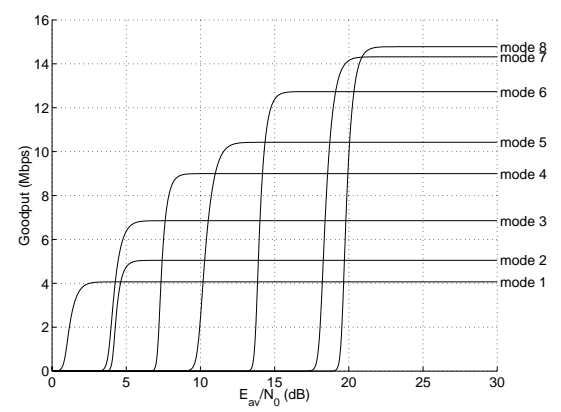

(b) 10 fragments

Fig. 6. Goodput vs. SNR

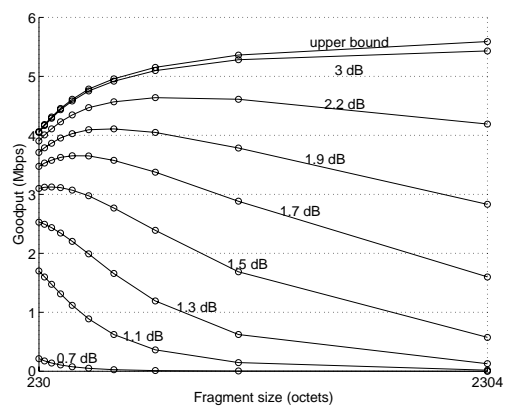

(a) PHY mode 1

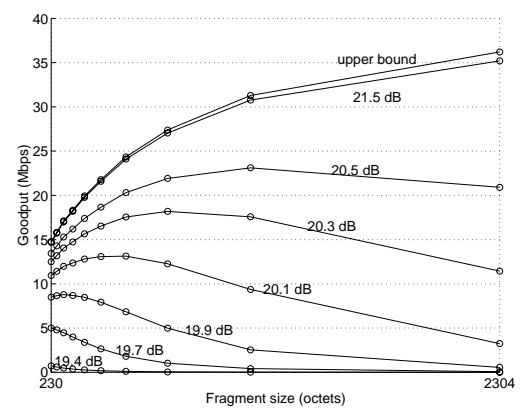

(b) PHY mode 8

Fig. 7. Goodput vs. Fragment size

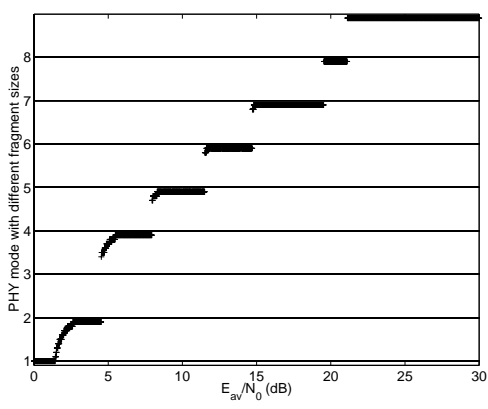

(a)

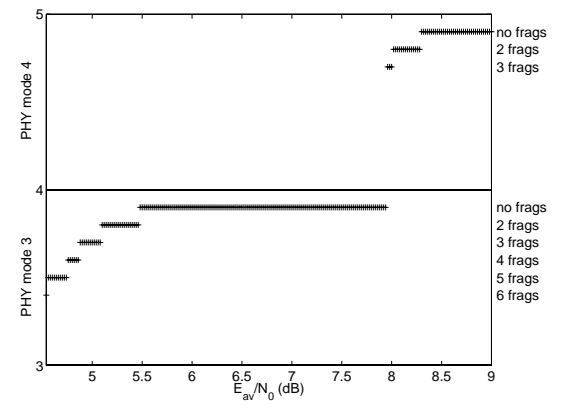

(b)

Fig. 8. Optimal PHY mode - fragment size combination for different SNRs

In this paper, we assume that the MSDUs to be transmitted are all of 2304-octet long, and each MSDU might be either not fragmented or fragmented to up to 10 equal-sized MPDUs. The numerical results of the goodput performance using different PHY modes and/or different fragment sizes are presented in the next subsection. Note that despite these assumptions, the results reflect the general trend.

\section{A. PHY Mode vs. Goodput}

The results of goodput performance for different PHY modes are shown in Fig. 6. As expected, the higher rate PHY modes show better goodput performance in the high SNR range, while the lower rate PHY modes have better goodput performance in the low SNR range. One interesting observation is that the goodput performance of PHY mode 3 (QPSK modulation with rate$1 / 2$ coding) is always better than that of PHY mode 2 (BPSK modulation with rate-3/4 coding) under all SNR conditions. The rationale behind is that, although QPSK has worse error performance than BPSK, the worse performance of the rate-3/4 convolutional code compared to the rate- $1 / 2$ convolutional code has the more dominating effect. Therefore, without the appropriate power control schemes, PHY mode 2 may not be a good choice at the presence of PHY mode 3. Another observation from Fig. 6 is that, for the same PHY mode, fragmentation decreases the maximum goodput due to the overheads, however, improves the goodput performance at certain SNR range. Please refer to PHY mode 5 at the SNR of $10 \mathrm{~dB}$ for an example.

Fig. 7 shows the goodput performance for different fragment sizes. The goodput upper bounds in the figure are obtained by assuming the error-free wireless channel, so the only factors to affect the goodput performance are the MAC and PHY layer 
overheads, and the ACK transmission time. We have three observations from this figure. First, for different fragment size, the goodput varies. Secondly, for different SNR condition, the optimal fragment size to achieve the best goodput performance varies. Thirdly, the affecting range of dynamic fragmentation is rather small compared to that by changing the PHY mode. The ranges for PHY mode 1 and PHY mode 8 are around $0.7 \sim 3 \mathrm{~dB}$ and 19.4 21.5 dB, respectively. Notice that, when PHY mode 8 is selected and when the SNR value is less than about $19 \mathrm{~dB}$, the goodput is almost zero, which means that, under low SNR conditions, the high rate 54 Mbps PHY mode is useless even with dynamic fragmentation.

The optimal combinations of the PHY mode and the fragment size to achieve the best goodput performance for different SNR conditions are shown in Fig. 8(a). Along the Y-axis, the values from $[y, y+1)$ corresponds to PHY mode $y$ with different fragmentation choices of no fragmentation to 10 fragments from up to down. For example, the selection of using PHY mode 1 with 10 fragments is shown as the lowest level in the figure. Fig. 8(b) is an enlarged version of Fig. 8(a) for the SNR range between $4.54 \mathrm{~dB}$ and $9 \mathrm{~dB}$. One observation from these optimal combinations is that, PHY mode 2 is not part of the optimal selection, which is consistent with the fact that PHY mode 2 has worse goodput performance than PHY mode 3 under all SNR conditions (see Fig. 6). Finally, Fig. 9 illustrates the maximum achievable goodput by choosing the optimal combination of the PHY mode and the fragment size, i.e., the optimal link adaptation, which is given in Fig. 8(a).

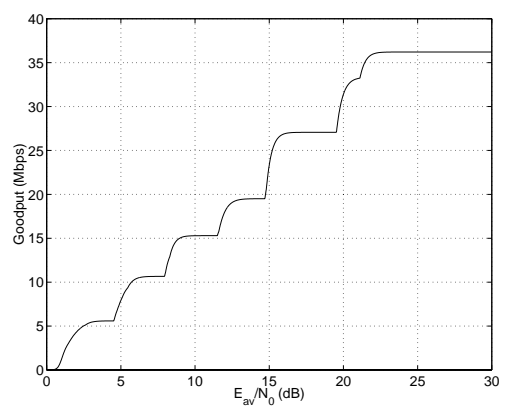

Fig. 9. Goodput upper bound from optimal link adaptation

\section{B. Link Adaptation}

Based on the above discussions, we suggest the use of link adaptation to improve the goodput performance of the IEEE 802.11 wireless LAN using the high-speed OFDM PHY. Fig. 10 shows a system architecture to adopt the link adaptation.

The link adaptor provides three levels of functionality. First, the link adaptor estimates the current SNR condition based on monitoring the channel conditions and the previous transmission results. Secondly, the link adaptor selects the optimal combination of the PHY mode and the fragment size based on the SNR estimation (see Fig. 8(a)), and sets the fragment size before an MSDU is fragmented. Thirdly, in the mid of an MSDU transmission, the link adaptor could change the PHY mode for the next MPDU transmission if there is any variation of the SNR condition, this is because that, once an MSDU has been fragmented, the fragment size will remain unchanged until the end of the transmission. The three functionalities are represented in

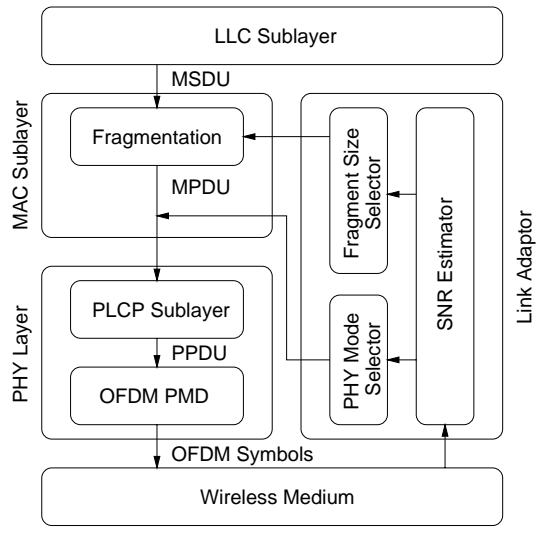

Fig. 10. System architecture for link adaptation

the figure as the SNR estimator, the fragment size selector, and the PHY mode selector, respectively.

One important aspect of this architecture is that, the implementation of the link adaptor is transparent to the higher layers, which makes it compatible to the existing network or higher layer applications. Besides, the basic idea of link adaptation is to take advantage of the different modulation schemes and FEC capabilities provided by the OFDM PHY, and no additional error correction codes need to be implemented. Therefore, the implementation of the link adaptor should be fairly simple, which makes this idea even more attractive.

\section{CONCLUSION}

In this paper, the key features of the DCF of the IEEE 802.11 MAC and the IEEE 802.11a OFDM PHY have been presented. The goodput performance is derived analytically for peer-topeer communication without interference in the IEEE 802.11 wireless LAN using the OFDM PHY. The numerical results suggest that link adaptation, which performs both dynamic fragmentation and PHY mode selection, is an attractive way to enhance the goodput performance of an IEEE 802.11a wireless LAN. In addition, a general system architecture to adopt link adaptation is also presented at the end of the paper.

As the future work, we are going to investigate the goodput performance in the multi-station environment through simulation, and design a link adaptation algorithm that can be embedded into the existing IEEE 802.11 MAC protocol.

\section{REFERENCES}

[1] IEEE 802.11 WG, Part 11: Wireless LAN Medium Access Control (MAC) and Physical Layer (PHY) specifications, Standard, IEEE, Aug 1999.

[2] IEEE 802.11 WG, Part 11: Wireless LAN Medium Access Control (MAC) and Physical Layer (PHY) specifications: High-speed Physical Layer in the $5 \mathrm{GHz}$ Band, Supplement to IEEE 802.11 Standard, Sep 1999.

[3] P. Lettieri and M. B. Srivastava, "Adaptive Frame Length Control for Improving Wireless Link Throughput, Range, and Energy Efficiency," in Proc. IEEE INFOCOM'98, San Francisco, California, Mar 1998.

[4] J. Khun-Jush, P. Schramm, U. Wachsmann, and F. Wenger, "Structure and Performance of the HIPERLAN/2 Physical Layer," in Proc. IEEE VTC'99 Fall, Amsterdam, Netherlands, Sep 1999.

[5] J. G. Proakis, Digital Communications, 3rd ed., McGraw Hill, New York, NY, 1995.

[6] M. B. Pursley and D. J. Taipale, "Error Probabilities for Spread-Spectrum Packet Radio with Convolutional Codes and Viterbi Decoding," IEEE Transactions on Communications, vol. COM-35, no. 1, pp. 1-12, Jan 1987.

[7] D. Haccoun and G. Begin, "High-Rate Punctured Convolutional Codes for Viterbi and Sequential Decoding," IEEE Transactions on Communications, vol. 37, no. 11, pp. 1113-1125, Nov 1989. 